\title{
An Accurate Estimation of the Eigenfrequency of an Offshore Wind Turbine Considered as the Stepped Euler-Bernoulli Beam in Three-Spring Flexible Foundation Using the Power Series Method
}

\author{
Mouafo Teifouet Armand Robinson $\mathbb{D}^{1,2}$ and Zhenyu Wang $\mathbb{D}^{1}$ \\ ${ }^{1}$ College of Civil Engineering and Architecture, Zhejiang University, Hangzhou 310058, China \\ ${ }^{2}$ Department of Physics, Faculty of Sciences, University of Dschang, PO-Box 0067, Dschang, Cameroon \\ Correspondence should be addressed to Mouafo Teifouet Armand Robinson; armandmouafo@yahoo.fr
}

Received 18 September 2021; Revised 6 November 2021; Accepted 9 November 2021; Published 7 December 2021

Academic Editor: Leopoldo Greco

Copyright (c) 2021 Mouafo Teifouet Armand Robinson and Zhenyu Wang. This is an open access article distributed under the Creative Commons Attribution License, which permits unrestricted use, distribution, and reproduction in any medium, provided the original work is properly cited.

\begin{abstract}
The present study employs the power series method (PSM) to accurately predict the natural frequencies of eleven offshore wind turbines (OWT). This prediction is very important as it helps in the quick verification of experimental or finite element results. This study idealizes the OWT as a stepped Euler-Bernoulli beam carrying a top mass and connected at its bottom to a flexible foundation. The first part of the beam represents a monopile and the transition piece while its second part is a tower. The foundation is modeled using three springs (lateral, rotational, and cross-coupling springs). This work's aim is at improving therefore the previous researches, in which the whole wind turbine was taken as a single beam, with a tower being tapered and its wall thickness being negligible compared to its diameter. In order to be closer to real-life OWT, three profiles of the tapered tower are explored: case 1 considers a tower with constant thickness along its height. Case 2 assumes a tower's thickness being negligible compared to its mean diameter, while case 3 describes the tower as a tapered beam with varying thickness along its height. Next, the calculated natural frequencies are compared to those obtained from measurements. Results reveal that case 2, used by previous researches, was only accurate for OWT with tower wall thickness lower than $15 \mathrm{~mm}$. Frequencies produced in case 3 are the most accurate as the relative error is up to $0.01 \%$, especially for the OWT with thicknesses higher or equal to $15 \mathrm{~mm}$. This case appears to be more realistic as, practically, wall thickness of a wind tower varies with its height. The towerto-pile thickness ratio is an important design parameter as it highly has impact on the natural frequency of OWT, and must therefore be taken into account during the design as well as lateral and rotational coupling springs.
\end{abstract}

\section{Introduction}

In the future, the offshore wind turbines are expected to become a significant contributor to electricity in China and in the rest of the world. They are generally designed to last 20 to 30 years at least. OWT face two major environmental forces coming from wind and water waves. The waves thus produced by sea water act on the OWT's substructure. The substructure can be the monopile, gravity-based structure, space frame structure (tripod, jacket, tripile), or floating structure. The most widely used substructure is the monopile, with the main advantage of being relatively easy and cheaper to manufacture and also easy to install. It accounts for $81 \%$ in the OWT manufacture industries up to date [1] and suitable for water depths of up to $30 \mathrm{~m}$. Its design remains fundamental in the construction of OWT because it undergoes the soil-structure interaction (SSI) effect [2].

Usually, three models of the soil-pile interaction are used: these are distributed springs (DS), coupled mode springs (CS), and apparent fixity (AF) [3].

The CS model represents the mostly used in the dynamic analysis of the offshore wind turbine as, it is applicable to all types of wind foundations and also easily implementable in theory. In this model, the foundation is modelled as a set 
of translational $\left(K_{L}\right)$, rotational $\left(K_{R}\right)$, and coupled $\left(K_{L R}\right)$ springs positioned at the bottom of the wind turbine. The values of those springs depend on the geometrical characteristics of the monopile and on the mechanical characteristics of the soil. Any change on those springs (soil nature due to the dynamic environment of the OWT) will have impact on the natural frequency of the OWT, which means that the natural frequency of the installed OWT could sometimes be greater or lower than the designed value and might also change over time [4]. As an illustration, the work published by Kallehave and Thilsted [5] has shown that the measured natural frequencies at the Walney site were reported to be 6-7\% higher than the designed values. The natural frequency appears therefore to be an important design calculation, and its decrease or increase with the time should be avoided as it may cause either unplanned resonance or increase the fatigue damage.

The whole OWT system is constituted by the pile, the tower, and the rotor-nacelle assembly. It could be simplified as a slender beam carrying a heavy top mass. This simplification helps on the quick calculation of the natural frequency of OWT without resort to the more accurate and expensive numerical model based on the finite element method. It concretely consists of constructing the mathematical model of the whole structure with the associated boundary conditions $([6,7])$, by considering the OWT as the Euler-Bernoulli beam [8] and then solving the obtained equation to get the natural frequencies. Similarly, Arany et al. [6] have worked on the analytical model to predict the natural frequency of offshore wind turbine using the Euler-Bernoulli and Timoshenko beam models. Four wind farms were considered, and the OWT was mathematically modelled as one slender beam with constant cross-section and constant thickness along the height. The obtained results were quite far from the measured values. They have also shown that modelling the OWT as the Timoshenko beam does not improve the accuracy of the natural frequency prediction. Later, Arany et al. [7] have used some simplified formulae to calculate the natural frequency of the wind turbine with acceptable accuracy. In fact, the tower was considered a tapered hollow cylinder with small wall thickness and the mean value of tower diameter was used. They analyzed the behavior of the natural frequency of ten wind turbines when the foundation flexibility changes and when the water depth varies.

In the present paper, an attempt is made to provide the natural frequency of OWT by using the power series method [9] and considering the system as a stepped Euler-Bernoulli beam $[10-21]$. The first part of the structure is constituted by the monopile and the transition piece, considered as a hollow cylinder [10] with constant wall thickness carrying springs at its bottom. The second part is constituted by the tower and modelled as a hollow cylinder with wall thickness varying with the height, with the heavy mass at the top. Three different mathematical formulations of the wall thickness of the tower are considered. The case producing the most accurate prediction of the natural frequency of the OWT will be highlighted. Also, the effect of the tower-to-pile wall thickness ratio and lateral and rotational springs on the natural frequencies will be discussed through some plots.

\section{The Model Description}

The offshore wind turbine system (Figure 1) in this paper is constituted by a monopile, the tower, and the rotor nacelle assembly (RNA). The total length of the system is $L$ while the first part's (pile + transition piece) length is $L_{p}$.

2.1. The Mathematical Model of OWT. Mathematically, the system is idealized as stepped beams with the first part (1) being constituted by the monopile and the transition piece and the second part (2) being constituted by the tower and the RNA (Figure 2). Real-life OWT might have a pile diameter equal to the tower bottom diameter $\left(D_{p}=D_{b}\right)$, greater $\left(D_{p}>D_{b}\right)$ or smaller than the tower bottom diameter $\left(D_{p}<D_{b}\right) . E, I$, and $A$ stand for the Young modulus, inertia, and cross-sectional area, respectively. $h$ is the wall thickness and $D$ is the diameter. Subscripts $p$ and $t$ stand for the pile and tower, respectively. $D_{b}$ and $D_{t}$ represent the tower bottom and the top diameter, respectively. $M$ and $J$ are the RNA mass and top mass moment of inertia, respectively. $P$ $(z)$ is the total axial force acting on the wind turbine structure.

2.2. The Foundation. The foundation is modelled using the coupled mode spring model which idealizes the foundation compliance as a set of translational and rotational coupled springs located at the mudline. In this analysis, the pile is slender and the soil is homogeneous. The whole structure is considered to be very stiff vertically, and consequently, only three springs are considered: the rotational $\left(K_{R}\right)$, translational $\left(K_{L}\right)$, and cross-coupling $\left(K_{L R}\right)$ spring. The approach developed by Gazeta [11] can be used to derive the values of the spring stiffness of the slender monopile. Eurocode 8 [12] could also be used when the soil is considered as homogeneous. Formulae developed by Pender [13] could apply for calculating the spring stiffness, taking into account the piles' Young's modulus and the bending stiffness ([14]). In matrix form, the three spring models are presented as follows:

$$
\left[\begin{array}{c}
F_{x} \\
M_{y}
\end{array}\right]=\left[\begin{array}{cc}
K_{L} & K_{L R} \\
K_{L R} & K_{R}
\end{array}\right]\left[\begin{array}{c}
w \\
w^{\prime}
\end{array}\right],
$$

with $F_{x}$ being the lateral force and $M_{y}$ being the fore-aft moment and $w$ and $w^{\prime}=\partial w / \partial x$ are the displacement and the slope, respectively.

2.3. The Axial Load. The tower weight appears to be higher than the nacelle weight for offshore wind turbines, and therefore, neglecting the tower mass may lead to erroneous results [15]. The top mass $M$ is constituted by the rotornacelle assembly with the mass moment of inertia $J$. The axial load is defined as the sum of the gravity force and self-weight of the structure. The value of the axial load depends on the height $z$ and is expressed through the 


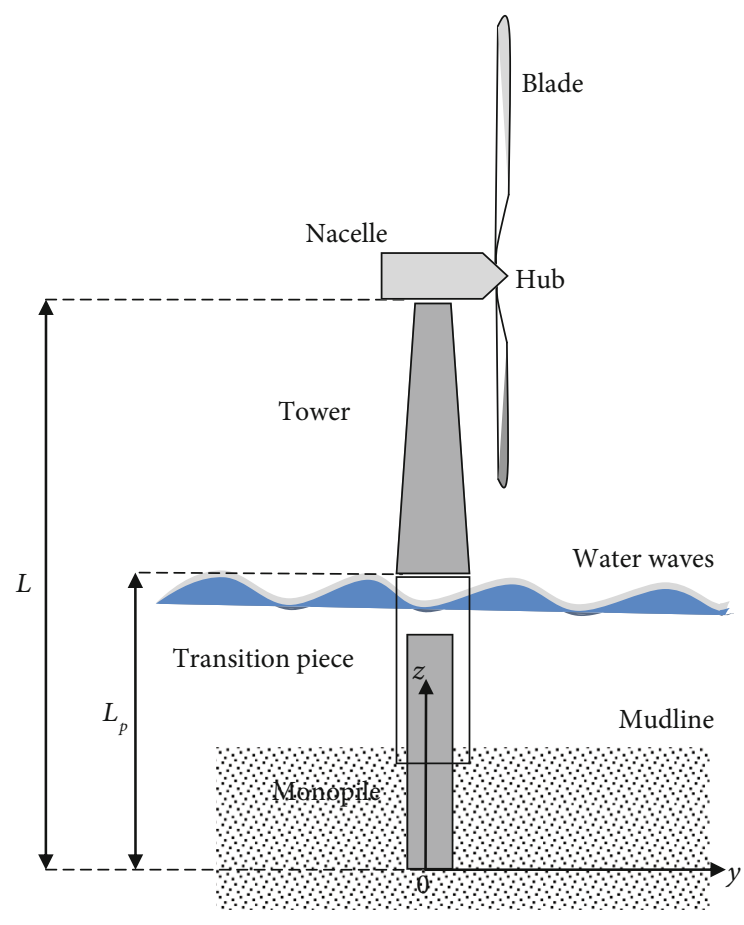

FIgURE 1: The offshore wind turbine.

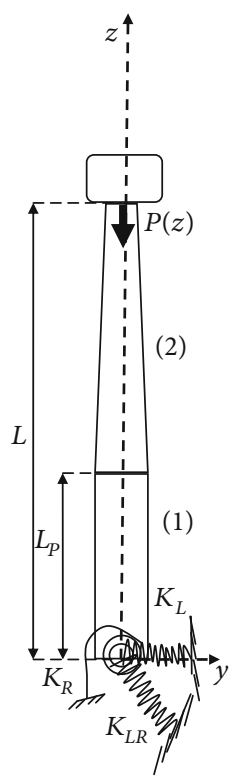

(a)

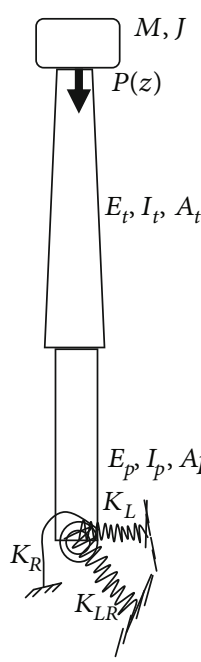

(b)

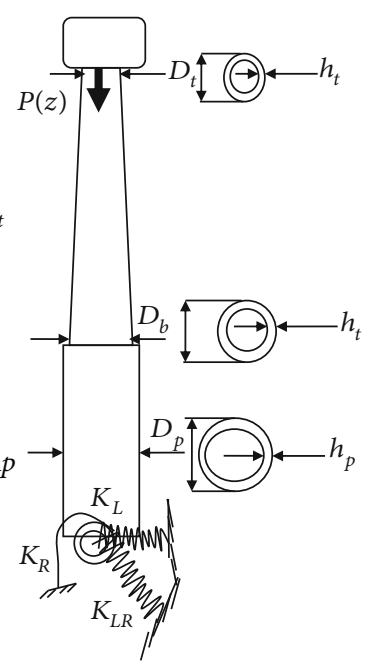

(c)
FIgure 2: Mathematical model of the OWT. (a) $D_{p}=D_{b}$. (b) $D_{p}$ $<D_{b}$. (c) $D_{p}>D_{b}$.

following formula [8]:

$$
P(z)=M g+\int_{z}^{L} \rho_{k} A_{k}(z) d z,
$$

where subscript $k=(p, t)$.
2.4. The Tapered Beam Profiles of the Tower. Generally, the tapered tower can be modeled by using three beam profiles as shown in Figure 3.

Figure 3(a) presents a tapered tower with a constant inner diameter and varying outer diameter along the height $z$ [16]. This form is not applied in the present paper as it requests that the bottom inner diameter should be smaller than the outer top diameter, but for all the studied wind turbine prototypes, $D_{i}>D_{t}$. The useful profiles are one those in Figures 3(b) and 3(c) summarized in the following three cases.

2.4.1. Case 1. This case models the wind turbine tower as a tapered beam with the circular cross-section. The outermost diameter $D_{o}$ and the innermost $D_{i}$ diameter linearly vary with respect to $z$ as follows:

$$
\begin{gathered}
D_{o}(z)=\left(\alpha_{o}+\beta_{o} z\right), \\
D_{i}=\left(\alpha_{i}+\beta_{i} z\right) .
\end{gathered}
$$

The area and inertia moment are derived as follows:

$$
\begin{aligned}
A_{t}(z) & =\frac{\pi}{4}\left[\alpha_{o}^{2}-\alpha_{i}^{2}+2\left(\alpha_{o} \beta_{o}-\alpha_{i} \beta_{i}\right) z+\left(\beta_{o}^{2}-\beta_{i}^{2}\right) z^{2}\right], \\
I_{t}(z)= & \frac{\pi}{64}\left[\left(\alpha_{o}^{4}-\alpha_{i}^{4}\right)+4\left(\alpha_{o}^{3} \beta_{o}-\alpha_{i}^{3} \beta_{i}\right) z+6\left(\alpha_{o}^{2} \beta_{o}^{2}-\alpha_{i}^{2} \beta_{i}^{2}\right) z^{2}\right. \\
& \left.+4\left(\alpha_{o} \beta_{o}^{3}-\alpha_{i} \beta_{i}^{3}\right) z^{3}+\left(\beta_{o}^{4}-\beta_{i}^{4}\right) z^{4}\right],
\end{aligned}
$$

where

$$
\begin{gathered}
\beta_{o}=\beta_{i}=D_{b} \frac{1-q}{L_{p}-L}, \\
\alpha_{0}=D_{b} \frac{q L_{p}-L}{L_{p}-L}, \\
\alpha_{i}=\alpha_{0}-2 h_{t} \quad q=\frac{D_{t}}{D_{b}} .
\end{gathered}
$$

Here, both the inner and outer diameters vary with $z$ but the wall thickness remains constant as shown in Figure 3(c).

2.4.2. Case 2. The tower is idealized as a hollow tapered beam (exactly like case 1) with very small wall thickness. The thin-walled approximation applies here and the diameters of tower are given as follows:

$$
\begin{gathered}
D_{o}(z)=D_{b}(\alpha+\beta z), \\
D_{i}=D_{o}-2 h_{t},
\end{gathered}
$$

followed by the area and inertia moment as follows:

$$
\begin{gathered}
A_{t}=\frac{\pi}{4}\left(D_{0}^{2}-\left(D_{0}-2 h_{t}\right)^{2}\right) \approx \pi D_{b} h_{t}(\alpha+\beta z)=A_{b}(\alpha+\beta z), \\
I_{t}=\frac{\pi}{64}\left(D_{0}^{4}-\left(D_{0}-2 h_{t}\right)^{4}\right) \approx \frac{\pi D_{b}^{3} h_{t}}{8}(\alpha+\beta z)^{3}=I_{b}(\alpha+\beta z)^{3},
\end{gathered}
$$




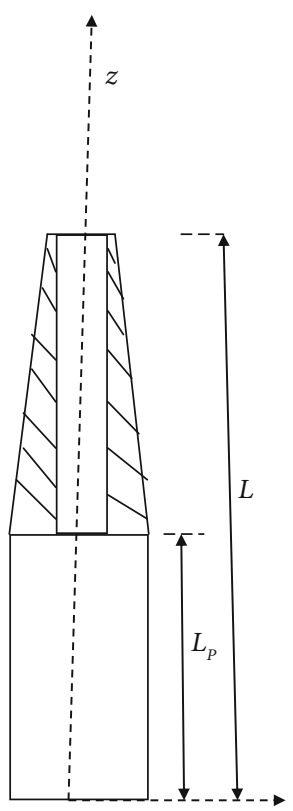

(a)

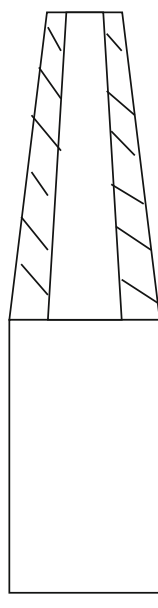

(b)

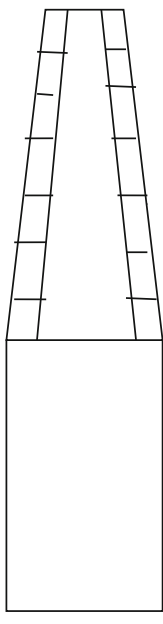

(c)

FIGURE 3: Mathematical model of OWT with three different forms of tapered tower.

with

$$
\begin{gathered}
\alpha=\frac{q L_{p}-L}{L_{p}-L}, \\
\beta=\frac{1-q}{L_{p}-L}, \\
q=\frac{D_{t}}{D_{b}} .
\end{gathered}
$$

2.4.3. Case 3. This case corresponds to Figure 3(b) where both the inner and outer diameter profiles are not parallel (as in Figure 3(c)). The wall thickness changes with the tower height [17]. This seems to describe more the reality than the two previous cases as the wind turbine tower is a sum of the welded tapered semicones with different wall thicknesses. The diameter, area, and inertia are given by the following formulae.

$$
\begin{gathered}
D_{o}(z)=D_{b}(\alpha+\beta z), \\
D_{i}(z)=\left(D_{b}-2 h_{t}\right)(\alpha+\beta z), \\
A_{t}(z)=\frac{\pi\left(D_{o}^{2}(z)-D_{i}^{2}(z)\right)}{4}=A_{b}\left[\alpha^{2}+2 \alpha \beta z+\beta^{2} z\right], \\
I_{t}(z)=\frac{\pi\left(D_{o}^{4}(z)-D_{i}^{4}(z)\right)}{64}=I_{b}\left[\alpha^{4}+4 \alpha^{3} \beta z+6 \alpha^{2} \beta^{2} z^{2}+4 \alpha \beta^{3} z^{3}+\beta^{4} z^{4}\right],
\end{gathered}
$$

with coefficients $\alpha$ and $\beta$ given in equation (8).

\section{Equations Governing the OWT}

The governing equations of the OWT are those of a stepped Euler-Bernoulli beam. The first line represented the vibra-
TABLE 1: Name and location of the considered offshore wind turbines.

\begin{tabular}{lc}
\hline No. & Wind name and location \\
\hline I & Lely offshore farm (Netherlands) \\
II & Irene Vorrink offshore wind farm (Netherlands) \\
III & Blyth offshore wind farm (UK) \\
IV & Kentish Flats offshore wind farm (UK) \\
V & Barrow offshore wind farm (UK) \\
VI & Thanet offshore wind farm (UK) \\
VII & Belwind 1 offshore wind farm (Belgium) \\
VIII & Burbo Bank offshore wind farm (UK) \\
IX & Walney 1 offshore wind farm (UK) \\
X & Gunfleet Sands offshore wind farm (UK) \\
XI & ZJU-P (China) \\
\hline
\end{tabular}

tion of part 1, considered as a hollow cylinder with constant thickness and constant cross-section while the second equation models the tower as a tapered beam with varying circular cross-section along its height. The mathematical formulation is $[8-16,18-21]$ as follows.

$$
\begin{aligned}
& \frac{\partial^{2}}{\partial z^{2}}\left(E_{p} I_{p} \frac{\partial^{2} w}{\partial z^{2}}\right)+\frac{\partial}{\partial z}\left(P(z) \frac{\partial w}{\partial z}\right)+\rho_{p} A_{p} \frac{\partial^{2} w}{\partial t^{2}}=0, \quad 0 \leq z \leq L_{p}, \\
& \frac{\partial^{2}}{\partial z^{2}}\left(E_{t} I_{t} \frac{\partial^{2} w}{\partial z^{2}}\right)+\frac{\partial}{\partial z}\left(P(z) \frac{\partial w}{\partial z}\right)+\rho_{t} A_{t} \frac{\partial^{2} w}{\partial t^{2}}=0, \quad L_{p}<z \leq L,
\end{aligned}
$$

where $w$ is the transverse displacement, $z$ is the coordinate axis along the height of the OWT, and $E_{t} I_{t}$ and $E_{p} I_{p}$ are 
TABLE 2: Characteristics of the ZJU-P offshore wind turbine.

\begin{tabular}{|c|c|c|c|}
\hline Wind parameters & Symbols & Units & ZJU-P (XI) \\
\hline (1) Rotor-nacelle assembly mass & $M$ & Ton & 218.28 \\
\hline (2) Total height of wind turbine & $L$ & $\mathrm{~m}$ & 106.67 \\
\hline (3) Tower bottom diameter & $D_{b}$ & $\mathrm{~m}$ & 3.16 \\
\hline (4) Tower top diameter & $D_{t}$ & $\mathrm{~m}$ & 5.5 \\
\hline (5) Tower Young's modulus & $E_{t}$ & $\mathrm{GPa}$ & 210 \\
\hline (6) First part height & $L_{P}$ & $\mathrm{~m}$ & 28 \\
\hline (7) Tower wall thickness & $h_{t}$ & $\mathrm{~mm}$ & 75 \\
\hline (8) Monopile diameter & $D_{p}$ & $\mathrm{~m}$ & 5.5 \\
\hline (9) Monopile wall thickness & $h_{p}$ & $\mathrm{~mm}$ & 75 \\
\hline (10) Monopile Young's modulus & $E_{t}$ & $\mathrm{GPa}$ & 210 \\
\hline (11) Lateral stiffness of foundation & $K_{L}$ & $\mathrm{GN} / \mathrm{m}$ & 0.206 \\
\hline (12) Cross-stiffness of foundation & $K_{L R}$ & GN & -3.08 \\
\hline (13) Rotational stiffness of foundation & $K_{R}$ & $\mathrm{GNm} / \mathrm{rad}$ & 83.3 \\
\hline
\end{tabular}

TABLE 3: Natural frequencies and comparisons.

\begin{tabular}{|c|c|c|c|c|c|c|c|}
\hline \multirow{3}{*}{ OWT name } & \multicolumn{7}{|c|}{ Natural frequency $(\mathrm{Hz})$ and error (\%) } \\
\hline & \multirow{2}{*}{ Measured frequency } & \multicolumn{2}{|c|}{ Case 1} & \multicolumn{2}{|c|}{ Case 2} & \multicolumn{2}{|c|}{ Case 3} \\
\hline & & Frequency & Error & Frequency & Error & Frequency & Error \\
\hline I Lely A2 & 0.634 & 0.738 & 16.53 & 0.676 & 6.56 & 0.727 & 15.34 \\
\hline II Irene Vorrink & 0.546 & 0.629 & 15.31 & 0.562 & 2.87 & 0.603 & 10.51 \\
\hline III Blyth & 0.488 & 0.456 & -6.61 & 0.395 & -19.03 & 0.470 & -3.60 \\
\hline IV Kentish flats & 0.339 & 0.348 & 2.60 & 0.313 & -7.75 & 0.339 & 0.01 \\
\hline V Barrow & 0.369 & 0.360 & -2.41 & 0.300 & -18.56 & 0.361 & -2.08 \\
\hline VI Thanet & 0.370 & 0.346 & -6.57 & 0.281 & -23.97 & 0.351 & -5.19 \\
\hline VII Belwind & 0.372 & 0.378 & 1.61 & 0.322 & -13.49 & 0.378 & 1.34 \\
\hline VIII Burbo Bank & 0.292 & 0.295 & 0.96 & 0.2704 & -7.39 & 0.292 & 0.07 \\
\hline IX Walney & 0.350 & 0.329 & -6.08 & 0.285 & -18.54 & 0.330 & -5.68 \\
\hline X Gunfleet Sands & 0.314 & 0.300 & -4.42 & 0.268 & -14.52 & 0.302 & -3.82 \\
\hline XI ZJU-P & 0.262 & 0.250 & -4.35 & 0.201 & -23.20 & 0.266 & 1.45 \\
\hline
\end{tabular}

the bending stiffness of the tower and monopile, respectively. $\rho_{p}$ and $\rho_{t}$ are the pile and tower densities, respectively. The presence of the top mass on the whole structure considered introduces the following boundary conditions at the top of the tower $(x=L)$ :

$$
\begin{gathered}
{\left[E_{t} I_{t} \frac{\partial^{2} w}{\partial z^{2}}+M e \frac{\partial^{2} w}{\partial t^{2}}+\left(J+M e^{2}\right) \frac{\partial^{2}}{\partial t^{2}} \frac{\partial w}{\partial z}\right]_{z=L}=0} \\
{\left[\frac{\partial}{\partial z}\left(E_{t} I_{t} \frac{\partial^{2} w}{\partial z^{2}}\right)+P(L) \frac{\partial w}{\partial z}-M \frac{\partial^{2} w}{\partial t^{2}}-M e \frac{\partial^{2}}{\partial t^{2}} \frac{\partial w}{\partial z}\right]_{z=L}=0}
\end{gathered}
$$

Likewise, the three-spring foundation at the bottom of the structure leads to an additional boundary condition as follows:

$$
\begin{gathered}
{\left[E_{P} I_{p} \frac{\partial^{2} w}{\partial z^{2}}\right]_{z=0}-K_{R} \frac{\partial w}{\partial z}(0, t)-K_{L R} w(0, t)=0,} \\
{\left[\frac{\partial}{\partial z}\left(E_{p} I_{p} \frac{\partial^{2} w}{\partial z^{2}}\right)+P(0) \frac{\partial w}{\partial z}\right]_{z=0}+K_{L} w(0, t)+K_{L R} \frac{\partial w}{\partial z}(0, t)=0,}
\end{gathered}
$$

where $P(0)$ is the axial force at $z=0$.

\section{Method of Solution}

The solution of equation (10) has the following form.

$$
w(z, t)=V(z) \exp (j \omega t)
$$

where $\omega$ is the vibration frequency and $V(z)$ stands as the 
mode shape. To determine $V(z)$, the first part with length $L_{p}$ has a displacement $V_{p}(z)$ and the second part with length $L-L_{p}$ is constituted by the tower and modeled as a tapered beam carrying the RNA at the top with displacement $V_{t}(z)$. $V(z)$ appears then as a piece-defined function along the structure defined as follows:

$$
V(z)=\left\{\begin{array}{cc}
V_{p}(z) & 0<z<L_{p} \\
V_{t}(z) & L_{p}<z<L .
\end{array}\right.
$$

Then, the previous equation (10) becomes

$$
\begin{aligned}
& \frac{\partial^{2}}{\partial z^{2}}\left(E_{p} I_{p} \frac{\partial^{2} V_{p}}{\partial z^{2}}\right)+\frac{\partial}{\partial z}\left(P(z) \frac{\partial V_{p}}{\partial z}\right)-\rho_{p} A_{p} \omega^{2} V_{p}=0, \quad 0 \leq z \leq L_{p}, \\
& \frac{\partial^{2}}{\partial z^{2}}\left(E_{t} I_{t} \frac{\partial^{2} V_{t}}{\partial z^{2}}\right)+\frac{\partial}{\partial z}\left(P(z) \frac{\partial V_{t}}{\partial z}\right)-\rho_{t} A_{t} \omega^{2} V_{t}=0, \quad L_{p}<z \leq L .
\end{aligned}
$$

The boundary conditions at $z=0$ is rewritten as follows:

$$
\begin{gathered}
{\left[E_{P} I_{p} \frac{\partial^{2} V_{p}}{\partial z^{2}}\right]_{z=0}-K_{R} \frac{\partial V_{p}}{\partial z}(0, t)-K_{L R} V_{p}(0, t)=0} \\
{\left[\frac{\partial}{\partial z}\left(E_{p} I_{p} \frac{\partial^{2} V_{p}}{\partial z^{2}}\right)+P(0) \frac{\partial V_{p}}{\partial z}\right]_{z=0}+K_{L} V_{p}(0, t)+K_{L R} \frac{\partial V_{p}}{\partial z}(0, t)=0 .}
\end{gathered}
$$

The top boundary condition becomes

$$
\begin{gathered}
{\left[E_{t} I_{t} \frac{\partial^{2} V_{t}}{\partial z^{2}}-M e \omega^{2} V_{t}+\left(J+M e^{2}\right) \omega^{2} \frac{\partial V_{t}}{\partial z}\right]_{z=L}=0} \\
{\left[\frac{\partial}{\partial z}\left(E_{t} I_{t} \frac{\partial^{2} V_{t}}{\partial z^{2}}\right)+P(L) \frac{\partial V_{t}}{\partial z}-M \omega^{2} V_{t}-M e \omega^{2} \frac{\partial V_{t}}{\partial z}\right]_{z=L}=0}
\end{gathered}
$$

Next, the power series method is employed to solve equations (15)-(17) [9-12]. It consists on choosing $V_{p}$ and $V_{t}$ as follows [18]:

$$
\begin{array}{lr}
V_{p}(z)=F_{1} V_{1 p}(z)+F_{2} V_{2 p}(z)+F_{3} V_{3 p}(z)+F_{4} V_{4 p}(z), & 0 \leq z \leq L_{p}, \\
V_{t}(z)=T_{1} V_{1 t}(z)+T_{2} V_{2 t}(z)+T_{3} V_{3 t}(z)+T_{4} V_{4 t}(z), & L_{p} \leq z \leq L,
\end{array}
$$

where $F_{n}$ and $T_{n}(n=1,2,3,4)$ stand for the constants and $V_{n f}$ and $V_{n t}$ are four linearly independent fundamental solutions, defined as follows.

$$
V_{n p}(z)=\sum_{i=0}^{\infty} a_{n i} z^{i}=a_{n 0}+a_{n 1} z+a_{n 2} z^{2}+a_{n 3} z^{3}+, \cdots,
$$

TABle 4: Comparison of case 3 data and results from Arany et al. [7].

\begin{tabular}{lccccc}
\hline & \multicolumn{5}{c}{ Natural frequency $(\mathrm{Hz})$ and error (\%) } \\
OWT name & Measured & $\begin{array}{c}\text { Case } \\
3\end{array}$ & Error & $\begin{array}{c}\text { Arany et al. } \\
{[8]}\end{array}$ & Error \\
\hline I Lely A2 & 0.634 & 0.727 & $\mathbf{1 5 . 3 4}$ & 0.643 & $\mathbf{1 . 3 6}$ \\
II Irene Vorrink & 0.546 & 0.603 & $\mathbf{1 0 . 5 1}$ & $0.552-0.555$ & $\mathbf{- 1 . 1 0}$ \\
III Blyth & 0.488 & 0.470 & $\mathbf{- 3 . 6 0}$ & 0.489 & $\mathbf{0 . 1 2}$ \\
IV Kentish & 0.339 & 0.339 & $\mathbf{0 . 0 1}$ & 0.339 & $\mathbf{0 . 0 1}$ \\
Flats & 0.369 & 0.361 & $\mathbf{- 2 . 0 8}$ & 0.367 & $\mathbf{- 0 . 5 4}$ \\
V Barrow & 0.370 & 0.351 & $\mathbf{- 5 . 1 9}$ & 0.382 & $\mathbf{3 . 0 8}$ \\
VI Thanet & 0.372 & 0.378 & $\mathbf{1 . 3 4}$ & 0.380 & $\mathbf{2 . 1 2}$ \\
VII Belwind & & & & & \\
VIII Burbo & 0.292 & 0.292 & $\mathbf{0 . 0 7}$ & 0.295 & $\mathbf{1 . 0 5}$ \\
Bank & 0.350 & 0.330 & $\mathbf{- 5 . 6 8}$ & 0.349 & $\mathbf{- 0 . 4 0}$ \\
IX Walney & & & & & \\
X Gunfleet & 0.314 & 0.302 & $\mathbf{- 3 . 8 2}$ & 0.315 & $\mathbf{0 . 3 1}$ \\
Sands & & & & & \\
XI ZJU-P & 0.262 & 0.266 & $\mathbf{1 . 4 5}$ & & \\
\hline
\end{tabular}

$$
V_{n t}(z)=\sum_{i=0}^{\infty} b_{n i} z^{i}=b_{n 0}+b_{n 1} z+b_{n 2} z^{2}+b_{n 3} z^{3}+, \cdots,
$$

with $a_{n i}$ and $b_{n i}$ being coefficients to be determined. Solutions (18)-(19) have eight unknowns $F_{n}$ and $T_{n}$, but only the following four boundary conditions are available. The power series formulations of the four boundary conditions (16)-(17) are obtained by introducing equations (18) and (19) into (17) considering equations (20)-(21) as follows:

$$
\begin{gathered}
2 E I_{p} a_{n, 2}-K_{R} a_{n, 1}-K_{L R} a_{n, 0}=0, \\
6 E I_{p} a_{n, 3}+\left(P(0)+K_{L R}\right) a_{n, 1}+K_{L} a_{n, 0}=0, \\
E I_{t}(L) \sum_{i=0}^{\infty}(i+1)(i+2) L^{i} b_{n, i+2}-\left(J+M e^{2}\right) \omega^{2} \sum_{i=0}^{\infty}(i+1) L^{i} b_{n, i+1} \\
-M e \omega^{2} \sum_{i=0}^{\infty} L^{i} b_{n, i}=0, \\
E I_{t}(L) \sum_{i=0}^{\infty}(i+1)(i+2)(i+3) L^{i} b_{n, i+3}+E I_{t}{ }^{\prime}(L) \sum_{i=0}^{\infty}(i+1)(i+2) L^{i} b_{n, i+2} \\
+\left(P(L)+M e \omega^{2}\right) \sum_{i=0}^{\infty}(i+1) L^{i} b_{n, i+1}+\omega^{2} M \sum_{i=0}^{\infty} L^{i} b_{n, i}=0,
\end{gathered}
$$

where $I_{t}^{\prime}(z)=d I_{t} / d z$.

Four additional boundary conditions are needed and will be obtained from the continuity relations at the separation zone between the two parts (i.e., at $z=L_{p}$ ). That continuity concerns the deflection $(V)$, the slope $(\varphi)$, moment $(M)$, and shear force $(Q)$. 
The power series formulations of those four continuity relations are given as follows:

$$
\begin{gathered}
V_{p}\left(L_{p}\right)=V_{t}\left(L_{p}\right), \quad \text { i.e., } \quad \sum_{i=0}^{\infty} a_{n i} L_{p}{ }^{i}-\sum_{i=0}^{\infty} b_{n i} L_{p}{ }^{i}=0, \\
\varphi_{p}\left(L_{p}\right)=\varphi_{t}\left(L_{p}\right), \quad \text { i.e., } \quad \sum_{i=0}^{\infty} i(i+1) a_{n, i+1} L_{p}{ }^{i}-\sum_{i=0}^{\infty} i(i+1) b_{n, i+1} L_{p}{ }^{i}=0, \\
M_{p}\left(L_{p}\right)=M_{t}\left(L_{p}\right), \quad \text { i.e., } \quad E I_{p} \sum_{i=0}^{\infty} i(i+1)(i+2) a_{n, i+2} L_{p}{ }^{i} \\
\quad-E I_{t}\left(L_{p}\right) \sum_{i=0}^{\infty} i(i+1)(i+2) b_{n, i+2} L_{p}{ }^{i}=0, \\
Q_{p}\left(L_{p}\right)=Q_{t}\left(L_{p}\right), \\
\text { i.e., }\left[E I_{p} \sum_{i=0}^{\infty}(i+1)(i+2)(i+3) \mathrm{a}_{n, i+3} L_{p}{ }^{i}+P\left(L_{p}\right) \sum_{i=0}^{\infty}(i+1) \mathrm{a}_{n, i+1} L_{p}{ }^{i}\right] \\
-\left[E I_{t}\left(L_{p}\right) \sum_{i=0}^{\infty}(i+1)(i+2)(i+3) b_{n, i+3} r_{l}{ }^{i}\right. \\
\left.+E I_{t}{ }^{\prime}\left(L_{p}\right) \sum_{i=0}^{\infty}(i+1)(i+2) b_{n, i+2} L_{p}{ }^{i}+P\left(L_{p}\right) \sum_{i=0}^{\infty}(i+1) b_{n, i+1} L_{p}{ }^{i}\right]=0 .
\end{gathered}
$$

From equations (22) and (23) the $8 \times 8$ matrix will be obtained. Setting the determinant of that matrix to zero will provide us with the natural frequency of the system.

$a_{n, i}$ and $b_{n, i}$ are recursive relations after introducing equations (18) and (19) into (15) considering equations (20)-(21). These recursive relations depend on the taper profile and are presented in details in the appendix.

\section{Results and Discussion}

In order to test the accuracy of the PSM on predicting the natural frequency of OWT, considered as the stepped Euler-Bernoulli beam, a total of eleven real-world wind turbine will be analyzed. This paper will provide the data of one wind turbine only. The data of the remaining ten OWT are presented in [7].

5.1. The Considered Parameters. The parameters considered in this paper are characteristics of ten OWT from different wind farms as described in [7]. Their numberings go from I to $\mathrm{X}$. The one supplementary data will be added, provided by our Zhejiang University Lab (ZJU-P), and will be identified by number XI, as shown in Table 1 as follows.

Next, the characteristics of OWT XI is presented in Table 2 as follows:

5.2. Result and Discussion. In order to test the accuracy of the power series method on predicting the natural frequencies of several wind turbines, three types of tapered tower were considered. The whole structure is considered as made by steel, and the first part of the structure is a hollow uniform cylinder. For each case, the PSM is implemented using $N=22$ terms in the series. The results from the three cases

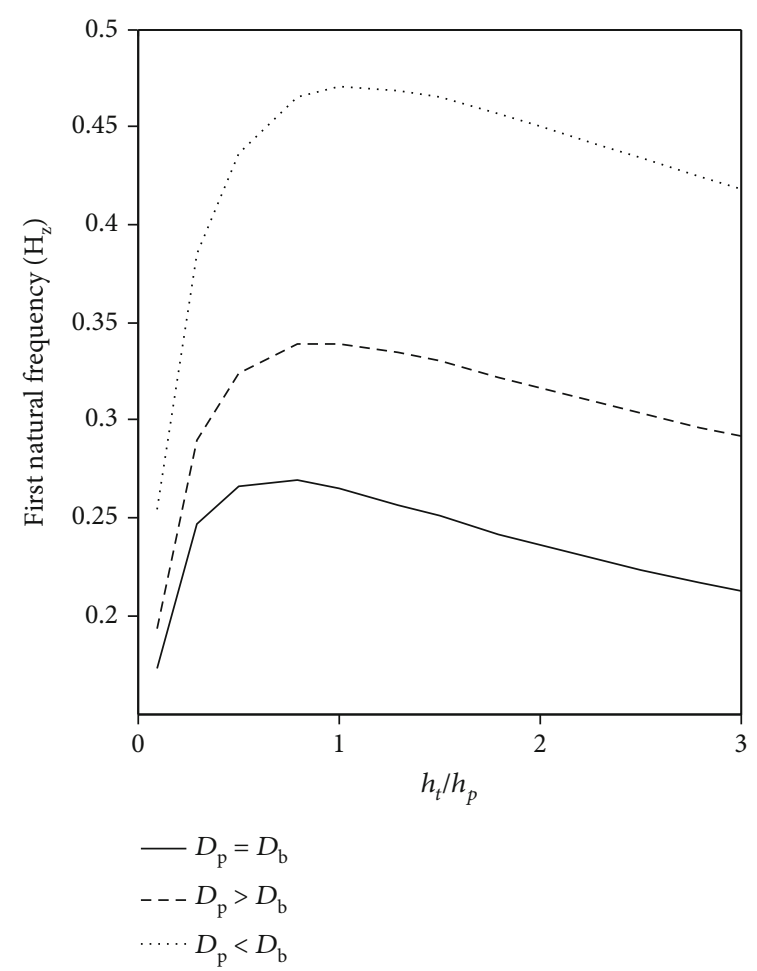

FIgURE 4: Natural frequency as a function of tower-to-pile thickness ratio.

are compared with the measured values as shown in Table 3. For each case, the relative error is calculated in order the appreciate the degree of accuracy.

For case 1, the profiles of the variation of the tower's inner and outermost diameters vary linearly along the height $z$ and remain parallel to each other. This implies that the wall thickness remains unchanged with the height $z$. When compared to the measured values, Lely A2 and Irene Vorrink show the very large discrepancy with the measured values. The relative errors for Blyth, Walney, and Thanet are about $6 \%$. The rest of OWT present an accuracy of about $4 \%$ at most.

For case 2, the tower is considered as having thin walls, and thus, its thickness can be neglected when compared to the bottom diameter $\left(h_{t} \ll D_{b}\right)$. The errors obtained for Lely A2 and Irene Vorrink OWT are $6.56 \%$ and $2.87 \%$, respectively, smaller, compared to $16.53 \%$ and $15.31 \%$, respectively, in case 1 . The rest of nine OWT show errors from 6.56 to $23.97 \%$. This approach appears as suitable only for I and II.

Case 3 supposes the thickness change along the tower height $z$ as well as outermost and innermost diameters. The obtained natural frequencies are quite close to those obtained by measurement except the frequencies of Lely A2 (15.34\%) and Irene Vorrink (10.51\%). This case increases the accuracy of all the frequencies, compared to those of case 1 .

Table 4 compares the case 3 results with those obtained by Arany et al. [7], and it is observable that the natural frequency of at Kentish Flats OWT is similar with measured 


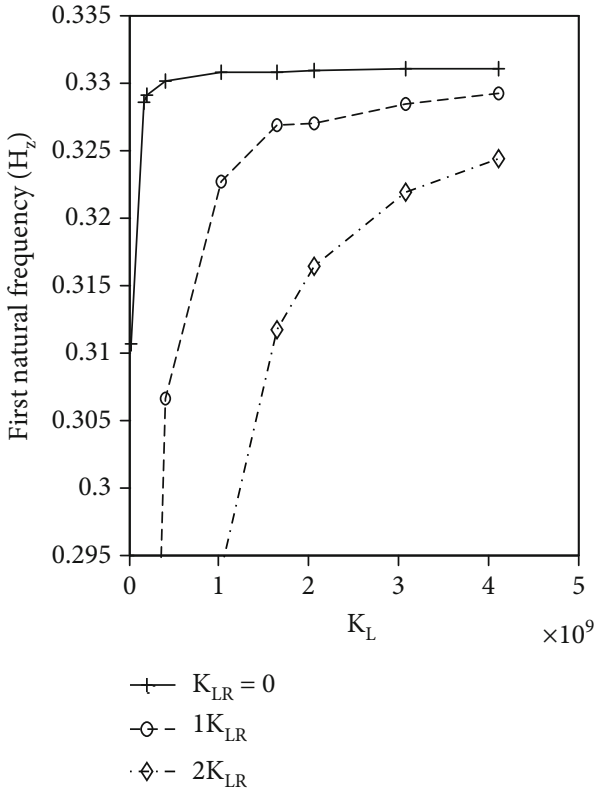

(a)

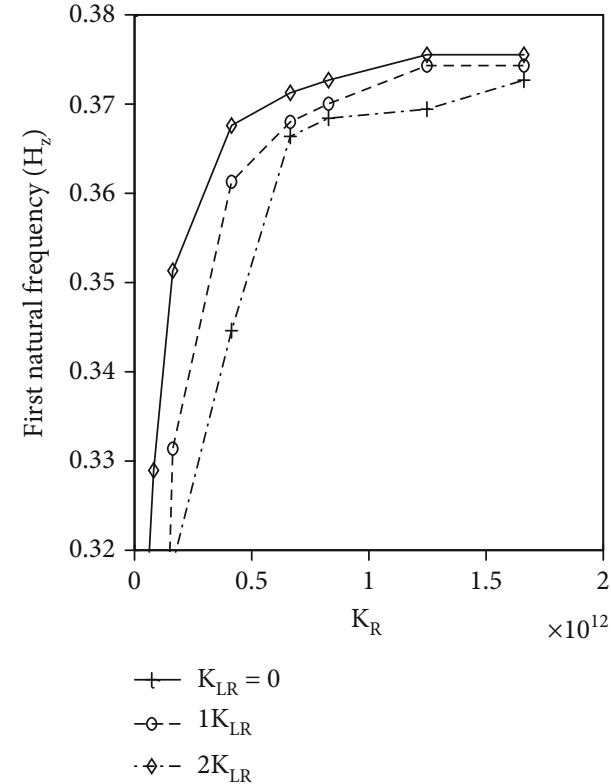

(b)

Figure 5: Natural frequency as a function of (a) lateral stiffness and (b) rotational stiffness.

value and the one obtained by Arany et al. [7]. Also, the present calculated Burbo Bank and Belwind natural frequencies are closer to the measured frequencies than those produced by Arany et al. [7]. For the rest, the discrepancy between the present results and measured results is that some thin-walled wind tower properties are not taken into account in the calculations. Also, the soil flexibility and water depth and added mass are not considered in our approach. The mass moment of inertia or the system damping was also not taken into account.

The natural frequencies of OWT appear to be highly sensitive to wall thickness, and this is the reason why Figure 4 was plotted, showing the frequency as a function of tower-to-pile thickness for three different configurations of the offshore wind turbine. ZJU-P $\left(D_{b}=D_{p}\right)$, Blyth $\left(D_{b}<D_{p}\right)$, and Walney I $\left(D_{b}>D_{p}\right)$ were chosen for the purpose, and the main observation is that the natural frequency increases with the tower-to-pile thickness ratio $h_{t} / h_{p}$, then reaches a maximum value, and starts decreasing. The maximum frequency is observed when $h_{t} / h_{p}=0.8$ for ZJU-P and $h_{t} / h_{p}=1$ for Blyth and Walney I. The decrease is slow for all three cases with the percentage around $11 \%$.

The behavior of frequency with lateral and rotational thickness was also examined in Figure 5 for different values of the cross-coupling spring for ZJU-P. Its observed in Figure 5(a) that independently to a chosen $K_{L R}$, the natural frequency of the structure increases when $K_{L}$ increases and then reaches an asymptotic value where the natural frequency becomes constant with when $K_{L R}$ increases, the asymptotic frequency decreases.

In Figure 5(b), the rotational spring appears to really have impact on the natural frequency as we observe a rapid increase of the natural frequency when $K_{R}$ increases. Then, an asymptotic frequency is reached earlier when $K_{L R}$ increases. $K_{R}$ has impact more on the natural frequency of OWT than $K_{L}$, as previously concluded in Arany et al. [7]

\section{Conclusion}

For the first time ever, the natural frequency of the offshore wind turbine is accurately predicted by considering the whole structure as a stepped Euler-Bernoulli beam and employing the power series (exact) method. It should be noted that the choice of the Euler-Bernoulli beam theories is because, as said at the introduction, the other beam theories have not improved the accuracy of the natural frequencies.

The wind turbine structure is divided into two parts. The first part, constituted by the monopile and the transition piece, is taken as a hollow cylinder with constant crosssection and constant thickness, carrying three springs at its bottom. The second part is a tower considered as a hollow semicone with variable cross-section which carries a heavy top mass. This consideration is the first novelty of this work as previous attempts have approximated the beam-like part of the OWT structure (monopile, transition piece, and tower) as a single beam, although these parts have different geometrical characteristics. We came therefore to the following conclusions:

(i) In the case 1 approach, where the tower wall thickness varies with its height, the obtained natural frequencies have the maximum error of $16.53 \%$ and the minimum of $0.96 \%$. These errors are greater than those of case 2, except for OWT with thicknesses greater or equal to $15 \mathrm{~mm}$ 
(ii) The case 2 approach is suitable for modeling Lely A1 and Irene Vorrink wind turbines as their tower thickness is negligible when compared to their bottom diameters

(iii) The case 3 approach tends to accurately calculate the natural frequency of OWT for tower thicknesses greater or equal to $15 \mathrm{~mm}$, with the maximum error range of about $5.7 \%$ and the minimum of about $0.01 \%$. This approach is quite close to the real wind tower because, in practice, the tower is constituted by multiple hollow semicones with different thicknesses which are welded to produce a whole structure

The present results are important and constitute a novelty because we have been able to show that case 2 is more indicated for an offshore wind turbine with a tower thickness lower than $15 \mathrm{~mm}$. Case 3, where the tower thickness wall varies with its height, must be used for finding the natural frequencies of OWT when it comes to structures with big wall thickness, if we need results with high precision.

(iv) -Tower-pile thickness and rotational spring are important parameters in the design as they highly have impact on the natural frequency

(v) This new and more complete approach is indicated to accurately predict the natural frequency of OWT when we have just few information about soil properties and environment conditions surrounding the site where the OWT is erected

\section{Appendix}

\section{A.1. The Power Series Method for Determining the Eigenfrequencies for Three Types of Tapered Towers}

The power series method is explained here for the second part (tower) of the stepped structure only. The first part's coefficients could be derived accordingly. The power series coefficients for all three cases are given as follows.

A.1. Case 1. The power series coefficients are given by the following recursive relations.

$$
\begin{gathered}
i=o, \\
c_{4} b_{n, 4}=-\frac{c_{3}}{2} b_{n, 3}-\frac{\left(2 c_{21}+c_{22}\right)}{12} b_{n, 2}-\frac{c_{12}}{24} b_{n, 1}-\frac{c_{03}}{24} b_{n, 0}, \\
i=1, \\
c_{4} b_{n, 5}=-\frac{3 c_{3}}{5} b_{n, 4}-\frac{\left(6 c_{21}+c_{22}\right)}{20} b_{n, 3}-\frac{\left(3 c_{11}+c_{12}\right)}{30} b_{n, 2} \\
-\frac{\left(2 c_{02}+c_{03}\right)}{120} b_{n, 1}-\frac{c_{-12}}{120} b_{n, 0},
\end{gathered}
$$

$$
\begin{gathered}
b_{n, i+4}=-r_{3} b_{n, i+3}-r_{2} b_{n, i+2}-r_{1} b_{n, i+1}-r_{0} b_{n, i}-r_{-1} b_{n, i-1}-r_{-2} b_{n, i-2}, \\
\kappa=c_{4}(i+1)(i+2)(i+3)(i+4), \\
r_{3}=\frac{(i+1)(i+2)^{2}(i+3) c_{3}}{\kappa}, \\
r_{2}=\frac{(i+1)(i+2)\left[(i+1)(i+2) c_{21}+c_{22}\right]}{\kappa}, \\
r_{0}=\frac{\left[(i-1) i(i+1)(i+2) c_{01}+i(i+1) c_{02}+c_{03}\right]}{\kappa}, \\
r_{1}=\frac{(i+1)^{2}\left[i(i+2) c_{11}+c_{12}\right]}{\kappa}, \\
r_{-1}=\frac{\left[(i-1)(i+1) c_{-11}+c_{-12}\right]}{\kappa}, \\
r_{-2}=\frac{c_{-2}}{\kappa}
\end{gathered}
$$

where

$$
\begin{gathered}
c_{4}=\frac{E \pi}{64}\left(\alpha_{o}^{4}-\alpha_{i}^{4}\right), \\
c_{3}=\frac{E \pi}{16}\left(\alpha_{o}^{3} \beta_{o}-\alpha_{i}^{3} \beta_{i}\right), \\
c_{21}=\frac{3 E \pi}{32}\left(\alpha_{o}^{2} \beta_{o}^{2}-\alpha_{i}^{2} \beta_{i}^{2}\right), \\
c_{22}=P(0), \\
c_{11}=\frac{E \pi}{16}\left(\beta_{o}^{3} \alpha_{o}-\beta_{i}^{3} \alpha_{i}\right), \\
c_{12}=\frac{\rho g \pi}{4}\left(\alpha_{i}^{2}-\alpha_{o}^{2}\right), \\
c_{01}=E \frac{\pi}{64}\left(\beta_{o}^{4}-\beta_{i}^{4}\right), \\
c_{02}=\frac{\rho g \pi}{4}\left(\beta_{i} \alpha_{i}-\beta_{0} \alpha_{o}\right), \\
c_{-12}=-\omega^{2} \frac{\rho \pi}{2}\left(\beta_{o} \alpha_{o}-\beta_{i} \alpha_{i}\right), \\
c_{03}=-\omega^{2} \frac{\rho \pi}{4}\left(\alpha_{o}^{2}-\alpha_{i}^{2}\right), \\
c_{-11}=\frac{\rho g \pi}{12}\left(\beta_{i}^{2}-\beta_{o}^{2}\right), \\
\left.\mathcal{B}_{o}^{2}-\alpha_{i}^{2}\right) L+\left(\alpha_{o} \beta_{o}-\alpha_{i} \beta_{i}\right) L^{2} \\
\left.\left.-\alpha_{i}^{2}-\alpha_{o}^{2}\right) z-\left(\alpha_{i} \beta_{i}-\alpha_{o} \beta_{o}\right) z^{2}-\frac{\left(\beta_{i}^{2}-\beta_{o}^{2}\right)}{3} z^{3}\right]
\end{gathered}
$$

A.2. Case 2. The power series coefficients are given by the 
following recursive relations.

$$
\begin{gathered}
i=o, \\
c_{4} b_{n, 4}=-\frac{c_{3}}{2} b_{n, 3}-\frac{\left(2 c_{21}+c_{22}\right)}{12} b_{n, 2}-\frac{c_{12}}{24} b_{n, 1}-\frac{c_{01}}{24} b_{n, 0} \\
i \geq 1 \\
b_{n, i+4}=-r_{3} b_{n, i+3}-r_{2} b_{n, i+2}-r_{1} b_{n, i+1}-r_{0} b_{n, i}-r_{-1} b_{n, i-1} \\
\kappa=c_{4}(i+1)(i+2)(i+3)(i+4) \\
r_{3}=\frac{(i+1)(i+2)^{2}(i+3) c_{3}}{\kappa}, \\
r_{2}=\frac{(i+1)(i+2)\left[(i+1)(i+2) c_{21}+c_{22}\right]}{\kappa}, \\
r_{1}=\frac{(i+1)^{2}\left[i(i+2) c_{11}+c_{12}\right]}{\kappa}, \\
r_{0}=\frac{\left[i(i+1) c_{02}+c_{01}\right]}{\kappa}, \\
r_{-1}=\frac{c_{-1}}{\kappa},
\end{gathered}
$$

where

$$
\begin{gathered}
c_{4}=E I_{b} \alpha^{3}, \\
c_{3}=3 E I_{b} \alpha^{2} \beta, \\
c_{21}=3 E I_{b} \alpha \beta^{2}, \\
c_{22}=P(0), \\
c_{11}=E I_{b} \beta^{3}, \\
c_{12}=-\rho g A_{b} \alpha, \\
c_{01}=-\rho A_{b} \omega^{2} \alpha, \\
c_{02}=\frac{-\rho g A_{b} \beta}{2}, \\
c_{-1}=-\rho A_{b} \omega^{2} \beta, \\
P(z)=M g+\rho g A_{b}\left(\alpha L+\frac{\beta L^{2}}{2}-\alpha z-\frac{\beta z^{2}}{2}\right) .
\end{gathered}
$$

A.3. Case 3. The power series coefficients are given by the following recursive relations.

$$
\begin{gathered}
i=o, \\
c_{4} b_{n, 4}=-\frac{c_{3}}{2} b_{n, 3}-\frac{\left(2 c_{21}+c_{22}\right)}{12} b_{n, 2}-\frac{c_{12}}{24} b_{n, 1}-\frac{c_{03}}{24} b_{n, 0}, \\
i=1, \\
c_{4} b_{n, 5}=-\frac{3 c_{3}}{5} b_{n, 4}-\frac{\left(6 c_{21}+c_{22}\right)}{20} b_{n, 3}-\frac{\left(3 c_{11}+c_{12}\right)}{30} b_{n, 2} \\
-\frac{\left(2 c_{02}+c_{03}\right)}{120} b_{n, 1}-\frac{c_{-12}}{120} b_{n, 0},
\end{gathered}
$$

$i \geq 2$,

$$
\begin{gathered}
b_{n, i+4}=-r_{3} b_{n, i+3}-r_{2} b_{n, i+2}-r_{1} b_{n, i+1}-r_{0} b_{n, i}-r_{-1} b_{n, i-1}-r_{-2} b_{n, i-2}, \\
\kappa=c_{4}(i+1)(i+2)(i+3)(i+4), \\
r_{3}=\frac{(i+1)(i+2)^{2}(i+3) c_{3}}{\kappa}, \\
r_{2}=\frac{(i+1)(i+2)\left[(i+1)(i+2) c_{21}+c_{22}\right]}{\kappa}, \\
r_{1}=\frac{\left[(i-1) i(i+1)(i+2) c_{01}+i(i+1) c_{02}+c_{03}\right]}{\kappa}, \\
r_{1}=\frac{(i+1)^{2}\left[i(i+2) c_{11}+c_{12}\right]}{\kappa}, \\
r_{-1}=\frac{\left[(i-1)(i+1) c_{-11}+c_{-12}\right]}{\kappa}, \\
r_{-2}=\frac{c_{-2}}{\kappa},
\end{gathered}
$$

where

$$
\begin{aligned}
& c_{21}=6 E I_{b} \alpha^{2} \beta^{2}, \\
& c_{22}=P(0), \\
& c_{11}=4 E I_{b} \beta^{3} \alpha, \\
& c_{12}=\rho g A_{b} \alpha^{2}, \\
& c_{01}=E I_{b} \beta^{4}, \\
& c_{02}=A_{b} \rho g \beta \alpha, \\
& c_{03}=-\omega^{2} \rho A_{b} \alpha^{2}, \\
& c_{-11}=\frac{\rho g A_{b}}{3} \beta^{2}, \\
& c_{-12}=-2 \omega^{2} \rho A_{b} \beta \alpha, \\
& c_{-2}=-\omega^{2} \rho A_{b} \beta^{2}, \\
& \alpha=\frac{q L_{p}-L}{L_{p}-L}, \\
& c_{4}=E I_{b} \alpha^{4}, \\
& c_{3}=4 E I_{b} \alpha^{3} \beta, \\
& P(z)=M g+\rho g A_{b}\left[\alpha^{2} L+\alpha \beta L^{2}+\frac{\beta^{2} L^{3}}{3}-\alpha^{2} z-\alpha \beta z^{2}-\frac{\beta^{2}}{3} z^{3}\right] .
\end{aligned}
$$

\section{Data Availability}

The data supporting the results can be found in the references mentioned inside the main text. 


\section{Conflicts of Interest}

The author declares no potential conflicts of interest with respect to the research, authorship, and/or publication of this article.

\section{Acknowledgments}

The authors gratefully acknowledge the financial support from the National Nature Science Foundation of China (nos. 51779224 and 51579221) and Zhejiang Provincial Natural Science Foundation of China (HZ19E090004).

\section{References}

[1] L. Ramirez, D. Fraile, and G. Brindley, "Offshore wind in Europe, key trends and statistics," 2020, https://windeurope .org/.

[2] S. Adhikari and S. Bhattacharya, "Vibrations of wind-turbines considering soil-structure interaction," Wind and Structures, vol. 14, no. 2, pp. 85-112, 2011.

[3] J. Jonkman and W. Musial, Offshore code comparison collaboration for IEA WindTask 23 offshore wind technology and deployment, National Renewable Energy Lab.(NREL), Golden, CO (United States), 2010.

[4] M. Kuhn, "Soft or stiff: a fundamental question for designers of offshore wind energy converters," in Proceding European Wind Energy Conference EWEC, Dublin Castle, Ireland, 1997.

[5] D. Kallehave and C. L. Thilsted, Modification of API -py formulation of initial stiffness of sand in offshore site investigation and geotechnics, Integrated Geotechnologies-Present and future, 2012.

[6] L. Arany, S. Bhattacharya, S. Adhikari, H. S. Hogan, and J. H. G. Macdonald, "An analytical model to predict the natural frequency of offshore wind turbines on three-spring flexible foundations using two different beam models," Soil Dynamics and Earthquake Engineering, vol. 74, pp. 40-45, 2015.

[7] L. Arany, S. Bhattacharya, J. H. G. Macdonald, and S. J. Hogan, "Closed form solution of Eigen frequency of monopile supported offshore wind turbines in deeper waters incorporating stiffness of substructure and SSI," Soil Dynamics and Earthquake Engineering, vol. 83, no. 1, pp. 18-32, 2016.

[8] M. Feyzollahzadeh, M. J. Mahmoodi, S. M. Yadavar-Nikravesh, and S. M. Jamali, "Wind load response of offshore wind turbine towers with fixed monopile platform," Journal of Wind Engineering and Industrial Aerodynamics, vol. 158, pp. 122138, 2016.

[9] M. T. Armand Robinson, T. Pierre Kisito, K. Y. Laurent, and S. Adali, "Analysis of the buckling of carbon nanotube under self-weight: the power series and differential quadrature approaches," Mechanics of Advanced Materials and Structures, vol. 26, no. 10, pp. 834-841, 2019.

[10] J. S. Wu and L. K. Chiang, "Free vibrations of solid and hollow wedge beams with rectangular or circular cross-sections and carrying any number of point masses," International. Journal of Numerical Method of Engineering, vol. 60, no. 3, pp. 695718, 2004.

[11] G. Gazeta, "Seismic response of end-bearing single piles," Journal of Soil Dynamics. Earthquake Engineering, vol. 3, no. 2, pp. 82-93, 1984.
[12] European Committee for standardization, Eurocode 8: Design of Structure of Earthquake Resistance -Part 5: Foundations, Retaining Structures and Geotechnical Aspects, European Commission, 2003.

[13] M. J. Pender, "Aseismic pile foundation design analysis," Bull New Zealand Society of Earthquake Engineering, vol. 26, no. 1, pp. 49-160, 1993.

[14] L. Arany, S. Bhattacharya, J. Macdonald, and S. J. Hogan, "Design of monopiles for offshore wind turbines in 10 steps," Engineering, vol. 92, pp. 126-152, 2017.

[15] B. Erica and M. Lance, "Foundation models for offshore wind turbines," in 47th AIAA Aerospace Sciences Meeting Including The New Horizons Forum and Aerospace Exposition, Florida, USA, 2009.

[16] Z. Wang and R. Li, "Transverse vibration of rotating tapered cantilever beam with hollow circular cross-section," Shock and Vibration., vol. 2018, pp. 1-14, 2018.

[17] M. T. A. Robinson and Z. Wang, "The effect of the TMD on the vibration of an offshore wind turbine considering three soil-pile-interaction models," Advances in Structural Engineering, vol. 24, no. 12, pp. 2652-2668, 2021.

[18] T. D. Chaudhari and S. K. Maiti, "A study of vibration of geometrically segmented beams with and without crack," International Journal of Solids and Structures, vol. 37, no. 5, pp. 761$779,2000$.

[19] R. D. Firouz-Abadi, M. Rahmanian, and M. Amabili, "Exact solutions for free vibrations and buckling of double tapered columns with elastic foundation and tip mass," ASME Journal of Vibration and Acoustic, vol. 135, no. 5, article 051017, 2013.

[20] D. Adair and M. Jaeger, "A power series solution for rotating nonuniform Euler-Bernoulli cantilever beams," Journal of Vibration and. Control, vol. 24, no. 17, pp. 3855-3864, 2018.

[21] X. Tong, X. Zhao, and S. Zhao, "Load reduction of a monopile wind turbine tower using optimal tuned mass dampers," International. Journal of Control, vol. 90, no. 7, pp. 1283-1298, 2017. 